\author{
Sebastian Rehberg \\ Christian Ertmer \\ Martin Westphal
}

\section{Valsalva, Valsalva, may you give me a clue, who needs fluids in my ICU?}

Accepted: 29 August 2008

Published online: 2 October 2008

(c) Springer-Verlag 2008

This editorial refers to the article available at: doi:10.1007/s00134-008-1295-1.

S. Rehberg · C. Ertmer · M. Westphal (৫)

Department of Anesthesiology and Intensive Care,

University Hospital of Muenster, Albert-Schweitzer-Str. 33,

48149 Münster, Germany

e-mail: martin.westphal@gmx.net

Tel.: +49-251-8347255

Fax: $+49-251-8348667$

Although described earlier by different physicians, the Valsalva maneuver (VM) was named after the Italian physiologist and surgeon Antonio Maria Valsalva, who defined this procedure in his work "De Aure Humana Tractatus" in 1704. However, at that time, it was mainly employed to expel foreign bodies or exsudates from the middle ear. Its use for diagnostic and investigative objectives is based on the detailed description of associated cardiovascular alterations by the German physiologist Edward Weber in 1851 [1].

These days, one of the most frequent challenges in the intensive care unit (ICU) is the judgement, whether or not a hemodynamically instable patient will profit from volume expansion in terms of an increase in arterial blood pressure. The decision is further complicated by the knowledge that fluid loading in non-responsive patients may not only delay effective therapy but even worsen outcome in ICU patients [2]. Various parameters have been proposed for the prediction of volume responsiveness in ventilated patients. In this regard, however, static measures of right and left ventricular preload, as well as end-diastolic ventricular dimensions, have a poor predictive value [3, 4]. Conversely, dynamic indices, such as stroke volume (SV) or SV-derived variables, e.g. aortic blood flow and pulse pressure variation $(\Delta \mathrm{PP})$, are more valuable to estimate the patients' volume status. But importantly, such variables have only been validated in mechanically ventilated patients and require the absence of cardiac arrhythmias or spontaneous breathing activity [5]. Therefore, the decision process is even more complicated in spontaneously breathing patients. So far, passive leg raising (PLR) represents the "gold standard" to predict volume responsiveness in spontaneously breathing patients, even in the presence of arrhythmias [6].

In the current issue of Intensive Care Medicine, Dr. García et al. report the results of a timely and innovative approach to establish the VM as a predictor of fluid responsiveness in spontaneously breathing ICU patients [7]. The VM was performed before and after an intravenous infusion of $500 \mathrm{~mL} \mathrm{6 \%}$ hydroxyethylstarch 130/0.4 (Voluven ${ }^{\circledR}$, Fresenius, Bad Homburg, Germany) in 30 patients with suspected hypovolemia. Volume-induced changes in SV indices were positively correlated with baseline values of Valsalva pulse pressure variation $(\triangle \mathrm{VPP})$ and Valsalva systolic pressure variation $(\Delta \mathrm{VSP})$. In addition, a correlation between the change in SV after volume resuscitation and the volume-induced changes in $\triangle$ VPP as well as $\triangle$ VSP was noticed.

An advantage of the VM over PLR is the direct correlation of $\triangle$ VPP with the SV. Physiologically, pulse pressure (PP) is correlated not only to SV but also to aortic compliance. However, since PPmax and PPmin are measured during the VM, aortic compliance is eliminated as a potential source of error. In the present study, sensitivity and specificity were highest for a $\triangle \mathrm{VPP}$ of $52 \%$ and a $\triangle$ VSP of $30 \%$, respectively. However, these $\Delta$-values are considerably high as compared to the positive predictive values of PLR (10\% for aortic blood flow and $12 \%$ for $\Delta \mathrm{PP})$. Since the impact of this difference on 
fluid management remains elusive, comparative future studies are needed to address this issue in more detail.

Both, PLR and VM, are simple, inexpensive and noninvasive transient procedures that may easily be performed in a few minutes and are related to minimal side effects. A disadvantage of the VM is the dependency on the active collaboration of the patient. Age, co-morbidities, current health condition and training of the patient may markedly influence the quality and the reliability of the measures. The correct performance of the VM, however, is even more important because of the major changes in $\triangle \mathrm{VPP}$ and $\Delta \mathrm{VSP}$ necessary to predict volume responsiveness. Unfortunately, the distribution of patient characteristics among the volume-responder and nonresponder groups is not mentioned in the manuscript by the current authors. In addition, the relatively high doses of vasopressor agents in four of the patients affect the response to dynamic tests [8]. For this reason, patients with catecholamine therapy should be investigated independently in future studies.

Although Dr. García and colleagues carefully provide evidence for the usefulness of the VM in clinical practice, some methodical limitations need to be taken into consideration. First, the authors state that the decision to give fluids was based on "the presence of hypotension, oliguria or tachycardia". However, hemodynamic data presented in table 2 neither show an increased heart rate, nor a decreased mean arterial pressure. In addition, no information is provided regarding urinary output. Therefore, it is difficult to transfer the present results to a specific patient population and to predict the usefulness in other patients, such as patients with severe arterial hypotension.

Second, the performance of the VM itself differs from the standard procedure. Although, several protocols are described in the literature, a strain over $20 \mathrm{~s}$ with an intraoral pressure of $40 \mathrm{mmHg}$ appears to be the most reliable and standardized form of the VM [9]. Whereas the average airway pressure of $33 \mathrm{cmH}_{2} \mathrm{O}$ (equivalent to $\sim 45 \mathrm{mmHg}$ ) is even higher than the standard procedure, the duration of $10 \mathrm{~s}$ is reduced by half of the time in the present study. A shorter duration of the VM may be appropriate to minimize the severity of side effects in ICU patients. However, a minimum of $15 \mathrm{~s}$ is recommended for high risk patients to achieve the necessary baroreflex response and marked modifications in autonomic activities [9]. This concern is supported by the observation that none of the two responder curves provided in the electronic supplement shows the expected decrease in HR during the VM or an increase thereafter.

In summary, the work of Dr. García and colleagues introduces a very interesting and feasible approach to predict volume responsiveness in spontaneously breathing patients. However, some practical and methodological issues need to be clarified, before drawing a final conclusion, whether Valsalva may give us a clue, who needs fluids in the ICU.

\section{References}

1. Derbes VJ, Kerr A Jr (1955) Valsalva's maneuver and Weber's experiment. $\mathrm{N}$ Engl J Med 253:822-823

2. Lowell JA, Schifferdecker C, Driscoll DF, Benotti PN, Bistrian BR (1990) Postoperative fluid overload: not a benign problem. Crit Care Med 18: 728-733

3. Coudray A, Romand JA, Treggiari M, Bendjelid K (2005) Fluid responsiveness in spontaneously breathing patients: a review of indexes used in intensive care. Crit Care Med 33:2757-2762
4. Teboul JL, Monnet X (2008) Prediction of volume responsiveness in critically ill patients with spontaneous breathing activity. Curr Opin Crit Care 14:334-339

5. Michard F, Teboul JL (2002) Predicting fluid responsiveness in ICU patients: a critical analysis of the evidence. Chest 121:2000-2008

6. Monnet X, Rienzo M, Osman D, Anguel N, Richard C, Pinsky MR, Teboul JL (2006) Passive leg raising predicts fluid responsiveness in the critically ill. Crit Care Med 34:1402-1407
7. Monge García MI, Gil Cano A, Díaz Monrové JC (2008) Arterial pressure changes during the Valsalva maneuver to predict fluid responsiveness in spontaneously breathing patients. Intensive Care Med. doi:10.1007/s00134-008-1295-1

8. De Backer D (2006) Can passive leg raising be used to guide fluid administration? Crit Care 10:170

9. Junqueira LF Jr (2008) Teaching cardiac autonomic function dynamics employing the Valsalva (Valsalva-Weber) maneuver. Adv Physiol Educ 32: $100-106$ 